\title{
SEPARATION OF VARIABLES ON A TRIANGULAR GEOMETRY
}

\author{
By \\ D. C. LOOK, JR. \\ University of Missouri-Rolla, Rolla, Missouri
}

\begin{abstract}
The usual separation of variables technique is used to obtain solutions on a triangular region of interest. The resulting error is noted and a forced orthogonality procedure is used to investigate the error. The forced method allows a means to indicate the onset of error based on the magnitude of two parameters.
\end{abstract}

1. Introduction. A common simplification made when analyzing an extended surface (fin or spline) is that the temperature variation within the fin is one-dimensional. Many papers (e.g., $[1,4,7,8]$ ) have shown this one-dimensional approach, although convenient, may cause error under certain physical conditions (e.g., when the convection coefficient $h$ for the fin surface is large compared to the fin material thermal conductivity $k$ ). Typically the validity criterion is described by the root Biot number magnitude $(\mathrm{Bi}=h l / k)$; for constant $h$, the one-dimensional assumption is that the Biot number, based on the half thickness of the fin root $(l)$, must be less than 0.1 . Most of the analytical works which produced this statement were carried out on one-dimensional and usually uniform cross-section fins.

Almost unnoticed have been two-dimensional analyses of fins (e.g.,[5]). Integral or numerical methods are used on all situations where the fin surfaces are not colinear with the geometry of the coordinate system (i.e, triangular fin in a rectangular or polar system). When the fin geometry and the coordinate geometry are colinear, the separation of variables method appears to be the accepted approach, except for a few exotic cases. On the other hand, several authors of partial differential equations books make statements precluding the use of the separation of variables approach in the instances of noncolinear coordinates and fin geometry. For example, in the book by Chester [2] the following statement is made: "Even if a PDE is separable in a given, say $\xi \eta$, coordinate system, the fact will be of no avail unless the BCs for the problem at hand are given only on coordinate curves."

This statement, of course, has a drastic impact on attempts to analyze nonuniform cross-section fins using a two-dimensional analysis. Thus the purpose of this note is to demonstrate the brute force application of the separation of variables method to analyze the temperature characteristics of a straight tapered or triangular fin, the problems encountered, and the identification of the onset of noticable error.

Received September 12, 1990.

Key words and phrases. Separation of variables.

(C) 1992 Brown University 
2. The problem. Consider the fin illustrated in Fig. 1. This is a side view of a very long triangular fin whose physical properties are constant; thus, a two-dimensional analysis seems appropriate. Note that a rectangular coordinate system is superimposed on the fin (the coordinate origin is at the center of the root). The wall temperature at $x=0$ is constant, as are the ambient fluid temperature $T_{\infty}$ and the fin material properties $(k, h)$.

Using the First Law of Thermodynamics and the Fourier-Biot Law as a basis for analysis, the two-dimensional conduction equation

$$
\frac{\partial^{2} \theta}{\partial x^{2}}+\frac{\partial^{2} \theta}{\partial y^{2}}=0
$$

may be deduced, where $\theta$ is the reduced temperature $T-T_{\infty}$. The corresponding boundary conditions for this geometry are:

$$
\begin{aligned}
x & =0, & T & =T_{W}, & \theta & =\theta_{W}, \\
x & =L, & \frac{\partial T}{\partial x} & =0, & \frac{\partial \theta}{\partial x} & =0, \\
y & =0, & \frac{\partial T}{\partial y} & =0, & \frac{\partial \theta}{\partial y} & =0,
\end{aligned}
$$

and

$$
\begin{gathered}
y=R(L-x), \\
-\left.\int_{0}^{l} \frac{\partial \theta}{\partial x}\right|_{x=0} d y=\frac{\mathrm{Bi}}{l} \int \theta d s=\frac{\mathrm{Bi} \sqrt{1+R^{2}}}{l R} \int_{0}^{l} \theta d y .
\end{gathered}
$$

Note that Eq.(2.3) is a force (insulation) condition at the tip, Eq. (2.4) is a symmetry condition, and Eq. (2.5) represents a steady-state condition on the tapered surface (i.e., the energy conducted into the fin at the root is convected from the tapered surfaces).

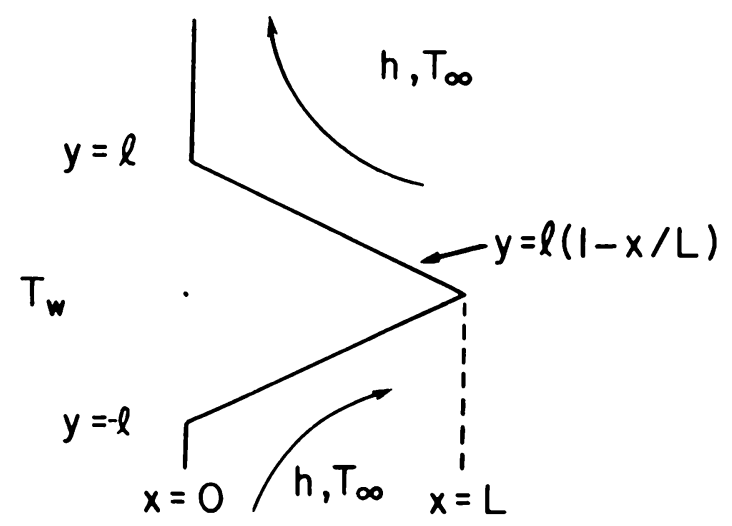

FIG. 1. Side view of two-dimensional fin. 
3. A solution. The boundary conditions require the solution to be

$$
\theta(x, y)=\sum_{n=1}^{\infty} B_{n} \operatorname{Cos}\left(\lambda_{n} y\right) \operatorname{Cosh}\left[\lambda_{n}(L-x)\right]
$$

where

$$
B_{n}=\frac{2 \theta_{W} \operatorname{Sin}\left(\lambda_{n} l\right)}{\lambda_{n} l\left[1+\operatorname{Sin}\left(2 \lambda_{n} l\right) / 2 \lambda_{n} l\right] \operatorname{Cosh}\left(\lambda_{n} L\right)} .
$$

Equation (2.5) (i.e., the boundary condition of Eq. (2.5)) yields the following equation when $x=\lambda_{n} l$ :

$$
\begin{aligned}
f(x ; R, \mathrm{Bi}) & =R \operatorname{Tan}(x)+\operatorname{Tanh}(x / R) \\
& -(x / \mathrm{Bi}) \sqrt{1+R^{2}} \operatorname{Tanh}(x / R) \operatorname{Tan}(x)=0 .
\end{aligned}
$$

4. The eigenvalue equation. Equation (3.3) is an unusual eigenvalue equation involving both orthogonal functions ( $\sin$ and $\cos$ ) and nonorthogonal functions (exponential). The variation of Eq. (3.3) for some sample values of the half width to length ratio $R$ and the parameter $\mathrm{Bi}$ is illustrated in Figs. 2 and 3 (see p. 144). The zeros of this function occur at the eigenvalues $\lambda_{n} l$. Perusal of these figures yields the conclusion that the zeros (the eigenvalues) are, with the exception of the first value, only a weak function of the parameter $\mathrm{Bi}$ and an even weaker function of $R$. The first eigenvalues, however, vary considerably with $\mathrm{Bi}$ and only slightly with respect to $R$.

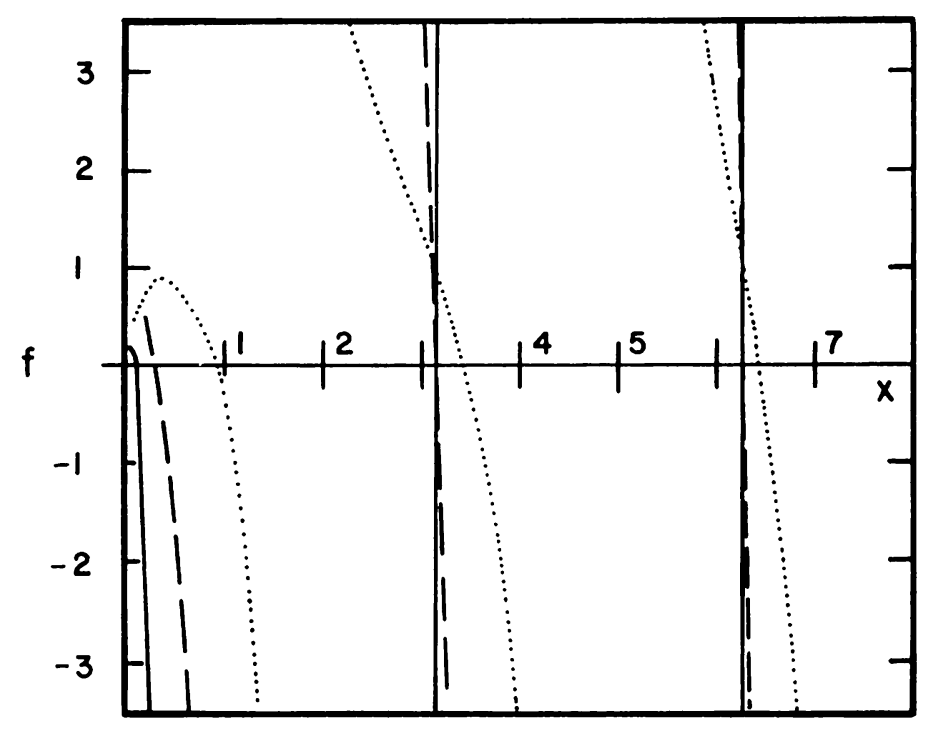

FIg. 2. The eigenvalue equation, $f(x ; R, \mathrm{Bi})$ versus $x\left(=\lambda_{n} l\right)$ for $R=0.2$ and $\mathrm{Bi}=0.01-, 0.1 \cdots$, and $1 \cdots \cdots$. 


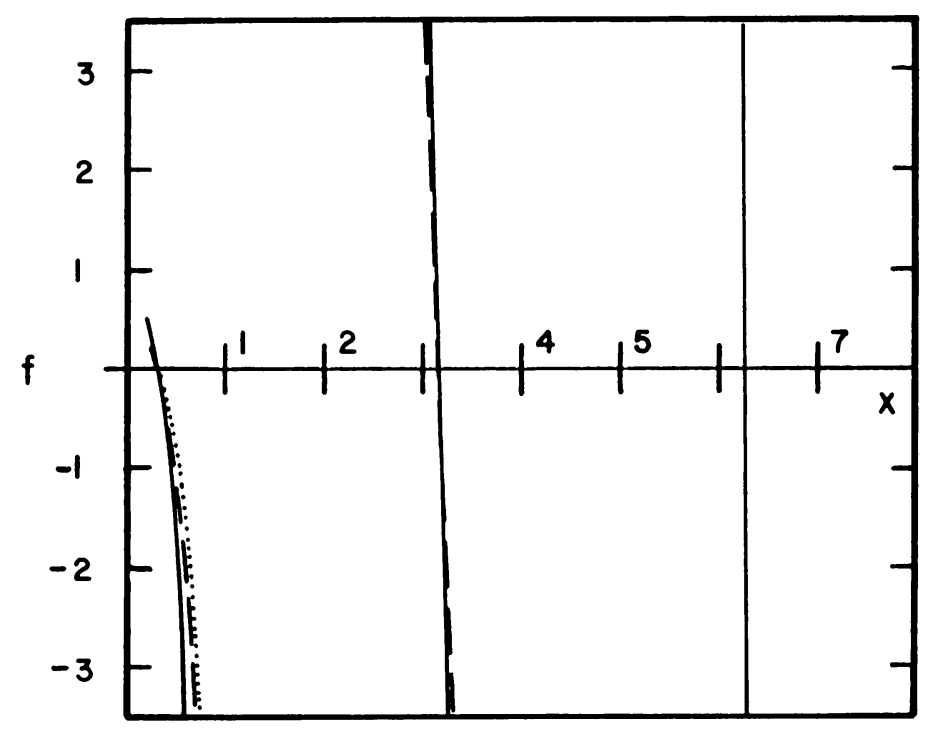

FIG. 3. The eigenvalue equation, $f\left(x_{i} ; R, \mathrm{Bi}\right)$ versus $x\left(=\lambda_{n} l\right)$ for $\mathrm{Bi}=0.1$ and $R=0.2-, 0.5 \cdots$, and $1 \cdots \cdot$.

5. Demonstration of results. The variation of the computed temperature profile $T(x, y=0)$ down the center of the fin from the root to the tip is illustrated in Fig.4. In order to obtain the solution, the summation indicated in Eq. (3.1) was continued at every point $x$ until the contribution of the next term was less than 0.00001 . The

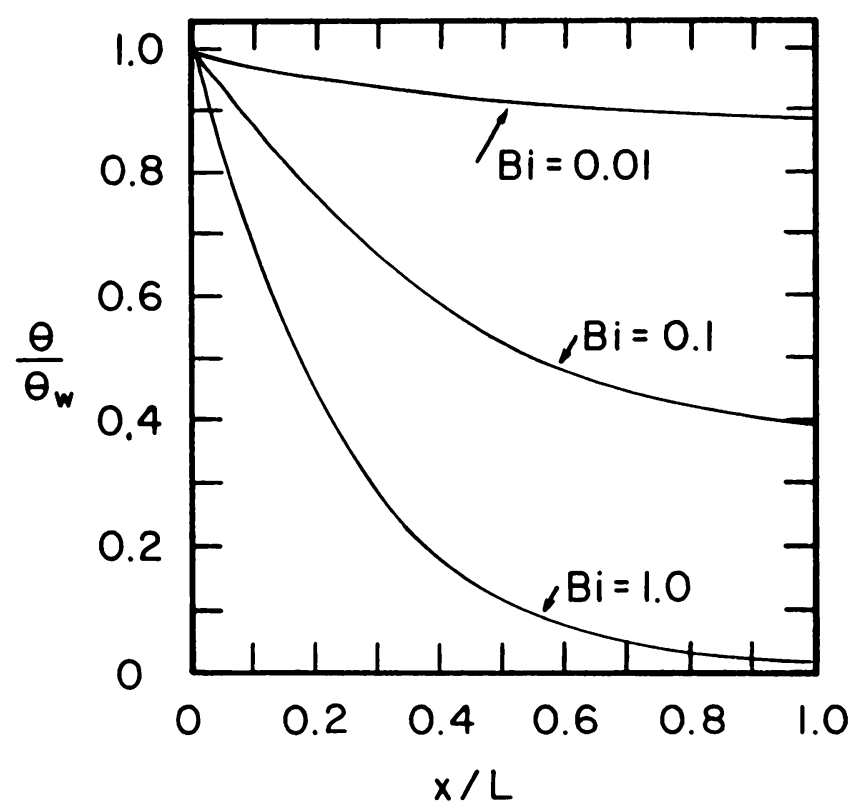

FIG. 4. Nondimensional temperature variation along the center line of a triangular fin for $R=0.2$ and $\mathrm{Bi}=0.01,0.1$, and 1.0 . 
Table I: Relative error in the temperature $T(x=0=y)$

at the center of the root

\begin{tabular}{l|l|l|l|}
$R$ & 0.01 & 0.1 & 1.0 \\
\hline 0.01 & 0 & 0 & 0.0006 \\
0.1 & 0 & 0.0005 & 0.0070 \\
0.2 & 0.0001 & 0.0010 & 0.0143 \\
0.5 & 0.0004 & 0.0039 & 0.0384 \\
1.0 & 0.0012 & 0.0117 & 0.0899 \\
2.0 & 0.0030 & 0.0295 & 0.1799
\end{tabular}

value of $R$ used is a working or typical value of the fin half width to fin length ratio while the values of $\mathrm{Bi}$ bracket the value traditionally accepted as the dividing line between using a fin $(\mathrm{Bi}<0.1)$ and not using a fin $(\mathrm{Bi}>0.1)$ in the one-dimensional case (e.g., $[3,4,6])$. All solutions seem to satisfy the boundary conditions at the tip. The condition at the root, on the other hand, is a different matter. A listing of the relative error at the center point of the root is given in Table I. Careful study of this table for the indicated range of the parameters $(R$ and $\mathrm{Bi})$ indicates that for $\mathrm{Bi} \leq 0.1$ and $R<1$, the error in the root temperature is minuscule. But as $\mathrm{Bi}$ and $R$ increase, the error increases dramatically.

6. The error. In the procedure used to obtain Eq. (3.2) an assumption is made. Usually without checking, it is assumed that for $\lambda \neq \lambda^{\prime}$,

$$
\int_{0}^{l} \operatorname{Cos}\left(\lambda^{\prime} y\right) \operatorname{Cos}(\lambda y) d y=\frac{\operatorname{Sin}\left[l\left(\lambda^{\prime}-\lambda\right)\right]}{2 l\left(\lambda^{\prime}-\lambda\right)}+\frac{\operatorname{Sin}\left[l\left(\lambda^{\prime}+\lambda\right)\right]}{2 l\left(\lambda^{\prime}+\lambda\right)}=0
$$

Unfortunately when using the first two zeros of Eq. (3.3), Eq. (6.1) is not satisfied. Thus the eigenvalue equation does not produce the appropriate characteristic values.

As a method of examination of the appropriate values, orthogonality may be "forced" by using Eq. (6.1). Thus $\lambda_{1} l\left(=x_{1}\right)$ was obtained from Eq. (3.3) and then used in Eq. (6.1) to obtain $\lambda_{2} l\left(=x_{2}\right)$ and so on. That is, the augmented form of Eq. (6.1),

$$
x_{i}=\left(2 x_{1}+x_{i}\right)-2\left(x_{i}+x_{1}\right) \frac{\operatorname{Tan}\left(x_{i}\right)}{\operatorname{Tan}\left(x_{i}\right)+\operatorname{Tan}\left(x_{1}\right)},
$$

was used in a trial and error procedure to obtain the other characteristic numbers. These results, besides satisfying Eq. (6.1), produce the correct result at the root re- 
Table II. Calculated temperature along the center of the fin using the usual and forced methods

\begin{tabular}{l|l|l|l|l|l|l|l} 
& \multicolumn{4}{|c|}{$T(x=0, y=0)$} & \multicolumn{2}{c|}{$T(x=0.1, y=0)$} & \multicolumn{2}{c}{$T(x=1, y=0)$} \\
$\mathrm{Bi}$ & $R$ & $\mathrm{U}$ & $\mathrm{F}$ & $\mathrm{U}$ & $\mathrm{F}$ & $\mathrm{U}$ & $\mathrm{F}$ \\
\hline 0.01 & 0.01 & 0.999997 & 0.999996 & 0.368929 & 0.368929 & 0.000092 & 0.000092 \\
& 0.1 & 1.000016 & 0.999995 & 0.929927 & 0.929926 & 0.647959 & 0.647959 \\
& 0.5 & 1.000379 & 1.000004 & 0.994814 & 0.996595 & 0.979864 & 0.979862 \\
& 1.0 & 1.001182 & 1.000004 & 1.000117 & 0.999212 & 0.995188 & 0.995067 \\
& 2.0 & 1.002983 & 1.000004 & 1.002332 & 0.999881 & 1.000592 & 0.999214 \\
\hline 0.1 & 0.01 & 1.000049 & 1.000004 & 0.045082 & 0.045082 & 0.000000 & 0.000000 \\
& 0.1 & 1.000456 & 1.000004 & 0.742095 & 0.742073 & 0.086810 & 0.086810 \\
& 0.5 & 1.003897 & 1.000004 & 0.972676 & 0.970387 & 0.826829 & 0.826813 \\
& 1.0 & 1.011712 & 0.999994 & 1.001394 & 0.992475 & 0.95381 & 0.952835 \\
& 2.0 & 1.029452 & 0.999987 & 1.024900 & 0.998711 & 1.005262 & 0.992123 \\
\hline 1.0 & 0.01 & 1.000637 & 0.999958 & 0.000198 & 0.000198 & 0.000000 & 0.000000 \\
& 0.1 & 1.006953 & 0.999953 & 0.455794 & 0.455596 & 0.000290 & 0.000290 \\
& 0.5 & 1.038366 & 0.999932 & 0.888024 & 0.868063 & 0.298741 & 0.298730 \\
& 1.0 & 1.089881 & 0.999888 & 1.011198 & 0.946909 & 0.674973 & 0.671675 \\
& 2.0 & 1.179878 & 0.999391 & 1.135434 & 0.985792 & 0.951044 & 0.909366
\end{tabular}

gardless of $\mathrm{Bi}$ and $R$. Table II is a listing of calculated temperatures at $x=0,0.1$, and 1 using the usual method $(U)$ and the forced method $(F)$. Oddly enough the solution using the usual and forced methods produces very similar results until both $\mathrm{Bi}$ and $R$ are large.

7. The reason. In order to discover the onset of failure, Eq. (3.3) needs to be rearranged to the form $\left(x_{n}=\lambda_{n} l\right)$

$$
x_{n} \operatorname{Tan}\left(x_{n}\right)=\left(\operatorname{Bi} / \sqrt{1+R^{2}}\right)\left[1+\left(R \operatorname{Tan}\left(x_{n}\right) / \operatorname{Tanh}\left(x_{n} / R\right)\right)\right]
$$

With effort, it can be shown that the solutions $\left(x_{n}\right)$ to Eq. (7.1) go very near as $n \pi$ for $n>2$. Thus only values of $x_{1}$ produce any real variation for changes in $\mathrm{Bi}$ and $R$. So for small $x_{1}$, Eq. (7.1) may be reduced to the approximate form

$$
x_{n} \operatorname{Tan}\left(x_{n}\right) \sim \operatorname{Bi} \sqrt{1+R^{2}} .
$$

Equation (7.2) is very similar to the eigenvalue equation encountered in the study of the thermal characteristic of uniform cross-section extended surfaces for which $R=0$. The characteristic values obtained using Eq. (7.1) and Eq. (7.2) for $R=0$ 


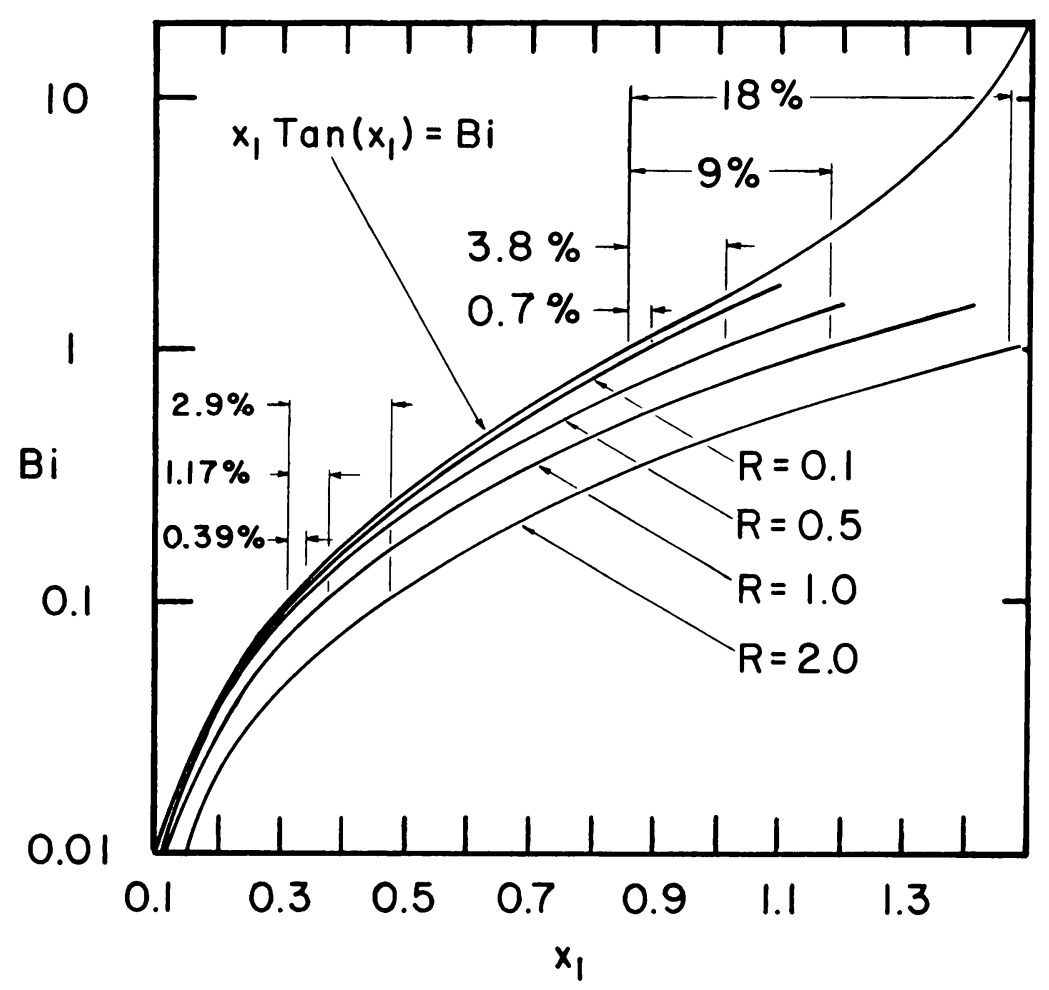

FIG. 5. Characteristic values produced by Eq. (7.2) for $R=0$ and those produced by Eq. (7.1) for various values of $R$. Also indicated are the percent error in the root temperature calculations in the cases of $\mathrm{Bi}=0.1$ and 1.0 for various values of $R$.

are illustrated in Fig. 5. It is noted that as $R$ increases, the values obtained from Eq. (7.1) deviate widely from those of Eq. (7.2) with $R=0$. This deviation is also a measure of the error in the center line root temperature. The approximate percent error of the root temperature is also indicated on Fig. 5. This error increases dramatically as $R$ increases beyond a value of 0.1 .

8. Conclusion. As is the conventional knowledge, the separation of variables method of solution is not an exact solution method when the coordinates and the system geometry are not colinear. What is particularly interesting is that there is a region for which apparently accurate solutions do exist. For the case presented here, the solutions have noticeable error at the root when $R$ is large (i.e., when the length of the fin becomes smaller-a bump on the surface). In fact, for the limited ranges of parameters used here, the error in the root temperature is very small for $R$ small (i.e., $R \leq 0.1$ ). Thus, while this limitation is bothersome, it is one that a fin designer might be able to work around. 


\section{REFERENCES}

[1] L. C. Burmeister, Triangular fin performance by the heat balance integral method, ASME J. Heat Transfer 101, 564-565 (1979)

[2] C. R. Chester, Techniques in Partial Differential Equations, McGraw-Hill, New York, 1971, p. 107

[3] E. R. G. Eckert and R. M. Drake, Jr., Analysis of Heat and Mass Transfer, McGraw-Hill, New York, 1972, p. 80

[4] R. K. Irey, Errors in the one-dimensional fin solution, ASME J. Heat Transfer 81, 151-156 (1968)

[5] W. Lau and C. W. Tan, Errors in one-dimensional heat transfer analysis in straight and annular fins, ASME J. Heat Transfer 95, 549-551 (1973)

[6] M. N. Özisik, Heat Transfer, a Basic Approach, McGraw-Hill, New York, 1985, p. 80

[7] A. D. Snider and A. D. Kraus, Recent developments in the analysis and design of extended surfaces, ASME J. Heat Transfer 105, 302-306 (1983)

[8] H. C. Unal, The effect of the boundary condition at a fin tip on the performance of the fin with and without generation, Internat. J. Heat Mass Transfer 31 (7), 1483-1496 (1988) 